\title{
INTRINSIC ALGEBRAS ON THE TORUS
}

\author{
BY \\ K. DE LEEUW AND H. MIRKIL
}

1. Introduction. An intrinsic homogeneous algebra $A$ on the $n$-dimensional torus $T_{n}$ is defined by the following properties:

(H0) $\boldsymbol{A}$ is a commutative semi-simple complex banach algebra.

(H1) The maximal-ideal space of $A$ is exactly the $n$-torus $T_{n}$.

(H2) $A$ is invariant with respect to all translations and with respect to all group automorphisms of $T_{n}$.

(H3) For each $f \in A$ the mapping $x \rightarrow f_{x}$ from $T_{n}$ to $A$ is continuous, $f_{x}$ being the translate of $f$ defined by $f_{x}(y)=f(y-x)$.

Condition $\mathrm{H} 1$ implies that multiplication is pointwise (not convolution), and that all $f \in A$ are continuous. In the presence of $\mathrm{H} 2$ and $\mathrm{H} 3$, condition $\mathrm{H} 1$ asserts equivalently that all real-analytic functions belong to $A$, i.e. all complex functions that possess near each $x \in T_{n}$ a convergent power series expansion in the $n$ real local coordinates. In particular $\boldsymbol{A}$ contains all constant functions. In the presence of $\mathrm{H} 1$ and $\mathrm{H} 2$, condition $\mathrm{H} 3$ asserts equivalently that the group characters all belong to $A$ and generate a dense subalgebra. For proofs of the above assertions see Silov [1] or Mirkil [1]. It is an easy consequence of $\mathrm{H} 3$ that the given norm on the algebra is uniformly bounded for all translations, hence we can assume that our norms are actually translation-invariant. It may not be true that the norm on $\boldsymbol{A}$ is uniformly bounded for all automorphisms of $\boldsymbol{T}_{n}$. In Šilov's work the invariance with respect to group automorphisms is omitted from $\mathrm{H} 2$, and the algebra $\boldsymbol{A}$ is simply called homogeneous.

In $\$ 3$ we prove that whenever an intrinsic homogeneous algebra $\boldsymbol{A}$ contains two different functions agreeing on one open subset of $\boldsymbol{T}_{n}$ then $\boldsymbol{A}$ contains functions separating any disjoint pair of closed subsets of $T_{n}$. In $\S 5$ we extend to the $n$-torus a theorem of Šilov concerning homogeneous algebras on the circle. In order to prove the theorem of $\S 5$ we must establish in $\S 4$ a purely algebraic theorem about ideals in formal power series invariant under unimodular substitutions.

2. Definitions and notations. We write the $n$-torus, even the circle, additively. We write $o$ for the identity element of $T_{n}$ (but 0 for the number zero, and $O$ for the zero element of a local algebra). It will of ten be convenient to choose a coordinate system so that each $x \in T_{n}$ is an $n$-tuple $\left(x_{1}, \cdots, x_{n}\right)$ of reals mod 1 . Relative to this coordinate system there are $n$ basic characters $e_{1}, \cdots, e_{n}$ defined by $e_{k}(x)=e_{k}\left(x_{1}, \cdots, x_{n}\right)=\exp \left(x_{k}\right)=\cos 2 \pi x_{k}+i \sin 2 \pi x_{k}$.

Received by the editors July $9,1955$. 
Every circle subgroup $T_{1}$ is a direct factor of $T_{n}$, hence a coordinate system can always be chosen to make $T_{1}=\left\{x \in T_{n}: x_{2}=x_{3}=\cdots \doteq x_{n}=0\right\}$.

It is easy to show that relative to a coordinate system the automorphisms of $T_{n}$ are represented by matrices with integer entries and determinant \pm 1 . Thus the only nontrivial automorphism of the circle $T_{1}$ is the reflection $x \rightarrow-x$. Throughout this paper we shall really use only the oriented automorphisms, i.e. those of determinant +1 . $T_{1}$ has none of these except the identity. Note that although the $n$-torus is a quotient of the $n$-dimensional vector group, the oriented automorphisms of the torus form a subgroup of the automorphisms of the vector group. This subgroup (=the integer matrices of determinant +1 ) is called the unimodular group.

Relative to a coordinate system there is a natural riemannian metric on $T_{n}$ that defines the square of the distance between $x$ and $y$ as the minimum of $\left(x_{1}-y_{1}+m_{1}\right)^{2}+\cdots+\left(x_{n}-y_{n}+m_{n}\right)^{2}$, taken over all $n$-tuples of integers $m_{1}, \cdots, m_{n}$. The metric is preserved when the coordinates $x_{k}$ are permuted and when certain $x_{k}$ are reflected into $-x_{k}$; these are in fact the only rigid automorphisms.

3. Quasi-analytic vs. regular. There exist certain homogeneous algebras, we shall call them quasi-analytic, which contain so few functions that the behavior of a function on any small open subset of the torus determines its values everywhere. Equivalently, if a function in a quasi-analytic algebra vanishes on an open set then it vanishes identically. Most known examples of such algebras are actually quasi-analytic in the stronger classical sense: they consist entirely of indefinitely-differentiable functions, and none of these functions (except 0 ) vanishes at a point together with all its derivatives. Denjoy, Carleman, and Mandelbrojt have made extensive study of this kind of quasi-analyticity. On the other hand, the homogeneous algebras of ordinary analysis (e.g. the continuous functions, the absolutely-convergent fourier series, the functions obeying a lipschitz condition, the differentiable functions) are the extreme opposite of quasi-analytic. Each of these algebras contains so many functions that any disjoint pair of closed sets on the torus can be separated by some function identically 0 on the first set, identically 1 on the second. Silov calls such algebras regular. The theorem below states that there are no homogeneous algebras of a sort midway between these extremes. "Quasi-analytic" is meant in our sense and not in the sense of DenjoyCarleman. The banach algebra expert will note that the proof would be simpler if $\boldsymbol{A}$ were closed under complex conjugation.

THEOREM 1. Every intrinsic homogeneous algebra $A$ on the $n$-torus $T_{n}$ must be either quasi-analytic or regular.

Proof. For each $f \in A$ define $Z(f)=\left\{x \in T_{n}: f(x)=0\right\}$, and let $Z^{0}(f)$ be the interior of $Z(f)$. Let $J=\left\{f \in A: o \in Z^{0}(f)\right\}$ and let $Z(J)=\cap Z(f)$, the intersection taken over all $f \in J$. By the general Šilov theory, see e.g. Mirkil [1], the 
regularity of $A$ is equivalent to the property $Z(J)=\{o\}$. The following lemma states that an even weaker property is sufficient.

Lemma A. If o is isolated in $Z(J)$, then $Z(J)$ contains no other points.

Proof of lemma. Let $U$ be an open neighborhood of $Z(J)$ such that the connected component of $U$ that contains $o$ contains no other points of $Z(J)$ and is far away from other components. For each $x \in U$ there exists $f_{x} \in J$ vanishing nowhere on some neighborhood $V_{x}$ of $x$. Finitely-many $V_{x}$ cover the (compact) complement of $U$. Let $W=\bigcap Z\left(f_{x}\right)$, for the $f_{x}$ corresponding to this finite number of $V_{x} . W$ is a closed neighborhood of $o$ contained in $U$, and for every $x$ not in $W$ there exists $f \in A, f \equiv 0$ on $W, f(x) \neq 0$. This is precisely the condition that the closed set $W$ be the maximal ideal space for the quotient algebra $\boldsymbol{A} / \boldsymbol{M}(W)$ obtained by restricting each $f \in A$ to $W$. Because of the way $U$ was chosen, $W$ is the union of disjoint closed subsets $W_{0}$ and $W_{1}$, with $W_{0} \cap Z(J)=\{0\}$ and $W_{0}$ open relative to $W$. Finitely-many characters $e_{1}, \cdots, e_{n}$ generate $\boldsymbol{A}$, hence their restrictions to $W$ generate $\boldsymbol{A} / \boldsymbol{M}(W)$. Define $S_{0}$ as the compact subset of complex $n$-space obtained by taking the direct product of the images $e_{1}\left(W_{0}\right), e_{2}\left(W_{0}\right), \cdots, e_{n}\left(W_{0}\right)$. Define $S_{1}$ similarly. $S_{0}$ and $S_{1}$ are disjoint. Let $\phi$ be a complex-analytic function $\equiv 0$ in some open neighborhood of $S_{0}$ and $\equiv 1$ in some disjoint neighborhood of $S_{1}$. Then the function $x \rightarrow \phi\left(e_{1}(x), \cdots, e_{n}(x)\right)$ belongs to $\boldsymbol{A} / \boldsymbol{M}(W)$. See Silov [4]. But this function is the restriction to $W$ of some $f \in J$ such that $Z(f) \cap Z(J)=\{0\}$. Hence $Z(J)=\{o\}$, and Lemma A is established.

Now suppose that $A$ is not quasi-analytic, so that $J$ contains some nontrivial function $g$. Define $S=\left\{x \in T_{n}: x+Z^{0}(g) \subset Z(g)\right\}$. $S$ is clearly a closed set stable under addition, hence it is a closed subgroup of $T_{n}$ and must be either finite or contain a circle $T_{1}$.

CASE I: $S$ finite. (Note that this must be the case when $T_{n}$ itself has dimension 1.) For $y \notin S$ we have $y+Z^{0}(g) \nsubseteq Z(g)$, and there exists $z \in Z^{0}(g)$ with $g(y+z) \neq 0$. But $g(y+z)=g_{-z}(y)$, and $g_{-z} \in J$. Hence $y \notin Z(J)$, and by Lemma A the algebra $A$ is regular.

CASE II: $S$ contains some circle $T_{1}$. ( $T_{n}$ has dimension $n \geqq 2$.) Express $T_{n}$ as the (internal) direct product of a circle $T_{1}$ and an $(n-1)$-torus $T_{n-1}$ so that each $x \in T_{n}$ can be written uniquely $x_{1}+\bar{x}$ with $x_{1} \in T_{1}$ and $\bar{x} \in T_{n-1}$. We proceed by induction on the dimension $n$. Take $\delta$ small enough so that $Z(g)$ contains the strip neighborhood $\left\{x \in T_{n}: x_{2}^{2}+x_{3}^{2}+\cdots+x_{n}^{2}<\delta\right\}$. For each $f \in A$, the following $A$-valued integral makes sense and defines a continuous projection $f \rightarrow f^{\sharp}$ of $A$ onto the subalgebra $A^{\sharp}$ of functions that do not depend on the first coordinate:

$$
f^{\sharp}=\int_{T_{1}} f_{x_{1}} d_{x_{1}} .
$$

The mapping $f \rightarrow f^{*}$ is of course not multiplicative. To prove it continuous, 
use the closed graph theorem, or calculate directly from the integral. Now let $e_{1}, \cdots, e_{n}$ be the basic characters relative to the given coordinate system on $T_{n}$. We can show that $e_{2}, \cdots, e_{n}$ generate $A^{\sharp}$. For let $f \in A$ and let $f_{n}$ approach $f$ in norm, where the $f_{n}$ are finite linear combinations of characters, i.e. polynomials in $e_{1}, \cdots, e_{n}$. Then $f_{n}^{*}$ approaches $f^{\sharp}$. But each $f_{n}$ is a linear combination of monomials like $e_{1}^{m_{1}} e_{2}^{m_{2}} \cdots e_{n}^{m_{n}}$, and

$\left(e_{1}^{m_{1}} \cdots e_{n}^{m_{n}}\right) \#(x)=\left(\int_{T_{1}} e_{1}^{m_{1}}(x) d x_{1}\right) e_{2}^{m_{2}}(x) \cdots e_{n}^{m_{n}}(x)$

with the integral of $e_{1}^{m_{1}}=0$ or 1 according as $m_{1} \neq 0$ or $=0$. If we consider $e_{2}, \cdots, e_{n}$ temporarily as functions on the maximal-ideal space $T^{*}$ of $A^{*}$ and let $y$ run over $T^{\sharp}$, then $y \rightarrow\left(e_{2}(y), \cdots, e_{n}(y)\right)$ is a homeomorphism of $T^{\sharp}$ into complex $(n-1)$-space. The image is called the "joint spectrum" of $e_{2}, \cdots, e_{n}$ with respect to $A^{\#}$. The ordinary spectrum of each $e_{k}(k \geqq 2)$ with respect to $A^{\#}$ is exactly what it was with respect to $A$, namely the complex numbers of absolute value one, because the inverse of a function independent of $x_{1}$ is also independent of $x_{1}$. Hence the above subset of complex $(n-1)$-spa:e is actually a subset of the direct product of $n-1$ unit circles. On the other hand, even with respect to $A$ the joint spectrum of $e_{2}, \cdots, e_{n}$ is already the full direct product of these circles. Hence each maximal ideal of $A^{A}$ is contained in an ideal of $\boldsymbol{A}$ and the maximal ideal space for $A^{\#}$ is the quotient space got by identifying all points in $T_{n}$ that differ only in their first coordinates. Interpreted as a homogeneous algebra on $T_{n-1}, A^{\#}$ must be either quasianalytic or regular by the induction assumption. Recall now that $Z(g)$ is known to contain an open strip, and let $h$ be a function in $A$ uniformly very close to the complex conjugate of $g$. Then $(h g)^{*} \in A^{\#}$ vanishes on an open strip but not identically, proving that $A^{\#}$ is regular. Given $x=\left(x_{1}, \cdots, x_{n}\right) \in T_{n}$ with $x_{k} \neq 0(k \geqq 2)$ there exists $f \in A^{\#}$ vanishing on a strip neighborhood of $o$, such that $f(x) \neq 0$. This proves that the ideal $J$ in $A$ has all its zeroes in the subgroup $T_{1}$. But $Z(J)$ is invariant under permutation of coordinates, hence $Z(J)=\{o\}$ and the regularity of $A$ is established.

4. Invariant ideals in formal power series. This section is purely algebraic, and can be read without reference to other sections. Throughout, $K$ will be an arbitrary field of characteristic 0 and $K\left[\left[X_{1}, \cdots, X_{n}\right]\right]$ will be the integral domain of formal power series in $n$ variables. The order of a formal power series is the degree of its lowest-degree term. The order of $O$ is often taken to be $+\infty$, just as the degree of $O$ is taken to be $-\infty$. Every $P \in K\left[\left[X_{1}, \cdots, X_{n}\right]\right]$ has order $\geqq 0$. The invertible $P$ are exactly those having order 0 , and the others form a unique maximal ideal $M$. (A commutative algebra that possesses exactly one maximal ideal is called a local algebra.) Under the natural valuation topology on $K\left[\left[X_{1}, \cdots, X_{n}\right]\right]$ defined by taking the powers of $\boldsymbol{M}$ as neighborhoods of $O$, series of high order are small and series of low order are large; thus the topology induced on $K$ is discrete. The 
polynomial algebra $K\left[X_{1}, \cdots, X_{n}\right]$ is a dense subalgebra of $K\left[\left[X_{1}, \cdots\right.\right.$, $\left.X_{n}\right]$ ] in this valuation topology, and in fact the formal power series become genuinely convergent power series. The only nontrivial fact about formal power series that we shall need is the following theorem.

THEOREM (KRULL). The valuation topology on $K\left[\left[X_{1}, \cdots, X_{n}\right]\right]$ is hausdorff and makes all ideals closed.

Proof. See Chevalley [1].

Lemma B. Every finite-dimensional local algebra $B$ over $K$ can be represented as a homomorphic image of $K\left[\left[X_{1}, \cdots, X_{n}\right]\right]$, for some $n$. The kernel of this homomorphism is an ideal containing some power of the unique maximal ideal $\boldsymbol{M}$ of $K\left[\left[X_{1}, \cdots, X_{n}\right]\right]$.

Proof. Let $Y_{1}, \cdots, Y_{n}$ be generators for the unique maximal ideal $N$ of $B$. Because $B$ is finite-dimensional some power $N^{r}$ is zero. A suitable homomorphism is that induced by $X_{1} \rightarrow Y_{1}, X_{2} \rightarrow Y_{2}$, etc., which takes $M \rightarrow N$ and hence takes $\boldsymbol{M}^{r} \rightarrow O$.

Lemma $\mathrm{B}$ above will not be used until the next section. But the lemmas below are needed in the present section for the proof of Theorem 2 .

Lemma C. Let $P_{1}, \cdots, P_{m}$ be linearly independent polynomials in $n$ variables over $K$. Let $v_{1}, \cdots, v_{m}$ span some vector space $V$ over $K$, and for integers $a_{1}, \cdots, a_{n}$ define the vector

$$
v\left(a_{1}, \cdots, a_{n}\right)=P_{1}\left(a_{1}, \cdots, a_{n}\right) v_{1}+\cdots+P_{m}\left(a_{1}, \cdots, a_{n}\right) v_{m} .
$$

Then as $a_{1}, \cdots, a_{n}$ run over all $n$-tuples of integers, the vectors $v\left(a_{1}, \cdots, a_{n}\right)$ also span $V$.

Proof. Suppose $v^{\prime}$ is a functional on $V$ orthogonal to all the vectors $v\left(a_{1}, \cdots, a_{n}\right)$ so that

$$
\begin{aligned}
\left\langle v\left(a_{1}, \cdots, a_{n}\right)\right| & \left.v^{\prime}\right\rangle \\
& =\left\langle\sum P_{i}\left(a_{1}, \cdots, a_{n}\right) v_{i} \mid v^{\prime}\right\rangle \\
& =\sum\left\langle v_{i} \mid v^{\prime}\right\rangle P_{i}\left(a_{1}, \cdots, a_{n}\right)=0 .
\end{aligned}
$$

Then the polynomial $\sum\left\langle v_{i} \mid v^{\prime}\right\rangle P_{i}$ vanishes on all integer $n$-tuples and must be identically zero. By the linear independence of the $P_{k}$ all the $\left\langle v_{k} \mid v^{\prime}\right\rangle$ are zero. Then $v^{\prime}$ can only be the zero functional, and the vectors $v\left(a_{1}, \cdots, a_{n}\right)$ must span $V$.

We shall say that a monomial really appears in a polynomial or power series if it has nonzero coefficient.

Lemma D. If $P \in K\left[X_{1}, \cdots, X_{n}\right]$ is homogeneous of degree $s \geqq 0$, then there exists some unimodular substitution $L$ such that $X_{1}^{s}$ really appears in $L(P)$.

Proof. The fact that $P\left(X_{1}, X_{2}, \cdots, X_{n}\right)$ does not vanish identically and 
is homogeneous guarantees that $P\left(1, X_{2}, \cdots, X_{n}\right)$ does not vanish identically. Let $b_{2}, \cdots, b_{n}$ be integers such that $P\left(1, b_{2}, \cdots, b_{n}\right) \neq 0$. Let $L$ be the unimodular substitution:

$$
\begin{aligned}
& X_{1} \rightarrow X_{1}, \\
& X_{2} \rightarrow b_{2} X_{1}+X_{2}, \\
& \cdot \cdot \cdot \cdot \cdot \cdot \cdot, \\
& X_{n} \rightarrow b_{n} X_{1}+X_{n} .
\end{aligned}
$$

Then the coefficient of $X_{1}^{s}$ in $L(P)$ is exactly $P\left(1, b_{2}, \cdots, b_{n}\right)$.

Lemma E. Let $\{P\}$ be a collection of polynomials in several variables such that the set $\{\Delta(P)\}$ is linearly independent, where $\Delta(P)$ is the homogeneous part of $P$ that has highest degree. Then the set $\{P\}$ is itself linearly independent.

Proof. Note that $\Delta\left(c_{1} P_{1}+c_{2} P_{2}\right)=c_{1} \Delta\left(P_{1}\right)+c_{2} \Delta\left(P_{2}\right)$ when $P_{1}$ and $P_{2}$ are of equal degree, but $\Delta\left(c_{1} P_{1}+c_{2} P_{2}\right)=c_{1} \Delta\left(P_{1}\right)$ when the degree of $P_{1}$ is higher. Consider some linear combination $c_{1} P_{1}+\cdots+c_{m} P_{m}=0$, with none of the scalars $c_{\boldsymbol{i}}$ zero. We may suppose the indices chosen so that $P_{1}, \cdots, P_{r}$ all have equal degree and $P_{r+1}, \cdots, P_{m}$ have various lower degrees. Then $0=\Delta\left(c_{1} P_{1}+\cdots+c_{m} P_{m}\right)=\Delta\left(c_{1} P_{1}+\cdots+c_{r} P_{r}\right)=c_{1} \Delta\left(P_{1}\right)+\cdots+c_{r} \Delta\left(P_{r}\right)$. But by the linear independence of $\{\Delta(P)\}$ we must have $c_{1}=c_{2}=\cdots=c_{r}=0$. This contradiction proves the lemma.

Lemma F. Let $m=$ the number of terms in the multinomial expansion of $\left(X_{1}+X_{2}+\cdots+X_{n}\right)^{s}$. If $P \in K\left[X_{1}, \cdots, X_{n}\right]$ is homogeneous of degree $s$, then there exist unimodular substitutions $L_{1}, \cdots, L_{m}$ such that $L_{1}(P), \cdots$, $L_{m}(P)$ span the vector space $K^{(s)}\left[X_{1}, \cdots, X_{n}\right]$ of homogeneous polynomials of degree $s$.

Proof. By Lemma D we can suppose $P=c X_{1}^{s}+Q$, with $X_{1}$ appearing in $Q$ to powers $<s$. For each $n$-tuple $\alpha=\left(a_{1}, \cdots, a_{n}\right)$ of integers with $a_{1}=1$, let $L_{\alpha}$ be the unimodular substitution defined by:

$$
\begin{aligned}
X_{1} & \rightarrow a_{1} X_{1}+\cdots+a_{n} X_{n}, \\
X_{2} & \rightarrow X_{2}, \\
\cdot & \cdot \cdot \cdot \cdot \cdot \cdot, \\
X_{n} & \rightarrow X_{n} .
\end{aligned}
$$

Let $X_{1}^{t_{1}} \cdots X_{n}{ }^{n}$ be one of the monomials (we ignore its coefficient) appearing in $Q$. By the definition of $Q, t_{2}+\cdots+t_{n}>0$. And the coefficient of $X_{1}^{s_{1}} \cdots$ $X_{n}^{s^{n}}$ in $L_{\alpha}\left(X_{1}^{t_{1}} \cdots X_{n}^{t^{n}}\right)=\left(X_{1}+a_{2} X_{2}+\cdots+a_{n} X_{n}\right)^{t_{1}} X_{2}^{t_{2}} \cdots X_{n}^{t^{n}}$ is a monomial in $a_{2}, \cdots, a_{n}$ of degree strictly less than $s-s_{1}$. Specifically, if $X_{1}^{s_{1}} \cdots X_{n}^{s_{n}}$ can appear at all it appears with coefficient $t_{1} !\left[s_{1} !\left(s_{2}-t_{2}\right) ! \cdots\left(s_{n}-t_{n}\right) !\right]^{-1}$

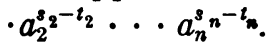


Hence the coefficient of $X_{1}^{s_{1}} \cdots X_{n}^{s_{n}}$ in all of $L_{\alpha}(Q)$ has degree strictly less than $s-s_{1}$. On the other hand, the coefficient of $X_{1}^{s_{1}} \cdots X_{n}^{s_{n}}$ in $L_{\alpha}\left(X_{1}^{s}\right)$ $=\left(X_{1}+a_{2} X_{2}+\cdots+a_{n} X_{n}\right)^{s}$ is of degree exactly $s-s_{1}$. Specifically this coefficient is $s !\left[s_{1} ! \cdots s_{n} !\right]^{-1} a_{2}^{s_{2}} \cdots a_{n}^{s_{n}}$. If for each $\sigma=\left(s_{1}, \cdots, s_{n}\right)$ we let $P_{\sigma}\left(a_{2}, \cdots, a_{n}\right)$ be the coefficient of $X_{1}^{s_{1}} \cdots X_{n}^{s_{n}}$ in $L_{\alpha}(P)=c L_{\alpha}\left(X_{1}^{s}\right)+L_{\alpha}(Q)$, then the term $a_{2}^{s_{2}} \cdots a_{n}^{s_{n}}$ really appears in $P_{\sigma}$ and all other terms of $P_{\sigma}$ have lower degree. Equivalently, each monomial $a_{2}^{s_{2}} \cdots a_{n}^{s_{n}}\left(s_{i}\right.$ integers $\geqq 0$ with $\left.s_{2}+\cdots+s_{n} \leqq s\right)$ really appears as highest degree term in exactly one of the $P_{\sigma}$. Thus by Lemma $\mathrm{E}$ the $P_{\sigma}$ form a linearly independent set.

Now we are in a position to apply Lemma C. We have $m$ linearly independent polynomials $P$ in $n-1$ variables, and a vector space $K^{(s)}\left[X_{1}, \cdots\right.$, $X_{n}$ ] spanned by the $m$ monomials $X_{1}^{s_{1}} \cdots X_{n}^{s_{n}}$. The conclusion of Lemma C then states that $K^{(s)}\left[X_{1}, \cdots, X_{n}\right]$ is spanned by homogeneous polynomials of the form $\sum_{\sigma} P_{\sigma}\left(a_{2}, \cdots, a_{n}\right) X_{1}^{s_{1}} \cdots X_{n}^{s_{n}}$. But this is also the conclusion of our present lemma, since

$$
\sum_{\sigma} P_{\sigma}\left(a_{2}, \cdots, a_{n}\right) X_{1}^{s_{1}} \cdots X_{n}^{s_{n}}=L_{\alpha}(P) .
$$

ThEOREM 2. Let $I$ be a proper ideal in $K\left[\left[X_{1}, \cdots, X_{n}\right]\right]$ invariant under unimodular substitutions. Then $I$ is some power of the unique maximal ideal $M$.

Proof. For each formal power series $P$ write $P^{(r)}$ for the homogeneous part of $P$ that has degree $r$, so that $P$ can be represented uniquely as an infinite sum

$$
P=P^{(0)}+P^{(1)}+P^{(2)}+\cdots .
$$

The mapping $P \rightarrow P^{(r)}$ is linear from $K\left[\left[X_{1}, \cdots, X_{n}\right]\right]$ to $K^{(r)}\left[X_{1}, \cdots, X_{n}\right]$. Choose $Q_{0} \in I$ with the property that $Q_{0}$ has order $s$ and no other element of $I$ has order less than $s$. By Lemma $F$ there exist unimodular substitutions $L_{1}, \cdots, L_{m}$ such that the $L_{i}\left(Q_{0}^{(s)}\right)$ span the vector space $\left.K_{0}^{(s+1)} X_{1}, \cdots, X_{n}\right]$ of homogeneous polynomials of degree $s$. Although we do not yet know that $I$ contains the $L_{i}\left(Q_{0}^{(s)}\right)$, we do know that $I$ contains the $L_{i}\left(Q_{0}\right)$ and linear combinations of them. In other words, for any $P^{(s)}$ homogeneous of degree $s$ there exists $Q \in I$ with $Q^{(s)}=P^{(s)}$.

We now claim that for any $P \in K\left[\left[X_{1}, \cdots, X_{n}\right]\right]$, and any integer $r>s$, there exists $Q \in I$ with $Q^{(s)}=P^{(s)}, Q^{(s+1)}=P^{(s+1)}, \cdots, Q^{(r)}=P^{(r)}$. For by induction we can at least choose some $Q_{0} \in I$ with $Q_{0}^{(s)}=P^{(s)}, Q_{0}^{(s+1)}=P^{(s+1)}, \cdots$, $Q_{0}^{(r-1)}=P^{(r-1)}$. (This $Q_{0}$ is of course different from the $Q_{0}$ in the paragraph above.) Let $R=P-Q_{0}$. Because $R$ has order $\geqq r$ we can write $R^{(r)}=X_{1} P_{1}$ $+X_{2} P_{2}+\cdots+X_{n} P_{n}$, with each $P_{i}$ either zero or homogeneous of degree $r-1$, though this expression for $R^{(r)}$ is not unique. Also by the induction assumption we know that $I$ contains $Q_{1}, \cdots, Q_{n}$ with $Q_{i}^{(r-1)}=P_{i}$ and hence $\left(X_{i} Q_{i}\right)^{(r)}=X_{i} P_{i}$. Let $Q=Q_{0}+X_{1} Q_{1}+\cdots+X_{n} Q_{n}$. For $s \leqq t \leqq r$ we have $Q^{(t)}=Q_{0}^{(t)}+\left(X_{1} Q_{1}+\cdots+X_{n} Q_{n}\right)^{(t)}$. If $t<r$ then $\left(X_{1} Q_{1}+\cdots+X_{n} Q_{n}\right)^{(t)}$ is zero because $X_{1} Q_{1}+\cdots+X_{n} Q_{n}$ has order $r$ (or possibly $\infty$ ), and $Q^{(t)}=Q_{0}^{(t)}$ 
$=P^{(t)}$. If $t=r$ then $\left(X_{1} Q_{1}+\cdots+X_{n} Q_{n}\right)^{(t)}=\left(X_{1} Q_{1}\right)^{(r)}+\cdots+\left(X_{n} Q_{n}\right)^{(r)}$ $=X_{1} P_{1}+\cdots+X_{n} P_{n}=R^{(r)}$, and $Q^{(t)}=Q_{0}^{(r)}+R^{(r)}=\left(Q_{0}+R\right)^{(r)}=P^{(r)}=P^{(t)}$. Hence our claim is established: for an arbitrary $P$ of order $\geqq s$, the ideal $I$ contains a sequence $\left\{Q_{r}\right\}$ such that $Q_{r}$ agrees with $P$ for all terms of degree $<r$. In other words $Q_{r}$ converges to $P$ in the valuation topology. To finish the proof of Theorem 2 what we need is exactly the theory of Krull cited above.

5. Homogeneous algebras of type $C$. We return to the intrinsic homogeneous algebra $\boldsymbol{A}$. Define $\boldsymbol{M}=\{f \in A: f(o)=0\}$ and define $J$ as in the proof of Theorem 1. $M$ is a maximal ideal. Throughout this section we shall suppose $\boldsymbol{A}$ regular, so that the ideal $J$ is contained in no maximal ideal except $\boldsymbol{M}$. In any algebra, an ideal contained in exactly one maximal ideal is called primary. $J$ is actually the smallest primary ideal in $\boldsymbol{M}$, and its closure $\bar{J}$ is the smallest closed primary ideal in $\boldsymbol{M}$. If we define $\boldsymbol{M}_{x}=\{f \in A: f(x)=0\}$ and $J_{x}$ as the smallest primary ideal in $\boldsymbol{M}_{x}$, then $\boldsymbol{M}_{x}$ and $J_{x}$ are simply translates of $\boldsymbol{M}$ and $J$. In particular, $\boldsymbol{M}=\boldsymbol{M}_{o}$ and $J=J_{o}$. The quotient algebra $\boldsymbol{A} / \bar{J}_{x}$ is a local banach algebra, in the purely algebraic sense of having only one maximal ideal, hence every ideal in $A / \bar{J}_{x}$ is primary. The elements of $A / \bar{J}_{x}$ are obtained by identifying $f_{1}$ and $f_{2}$ whenever they can be approximated in norm by functions agreeing near $x$. All the local algebras $A / \bar{J}_{x}$ are canonically isomorphic with $A / \bar{J}$ because translations of $T_{n}$ induce automorphisms of $A$.

This section will restrict itself to algebras of type $C$, in which by definition the norm of each $f \in A$ is the supremum of its quotient norms in the various local algebras: $\|f\|=\sup _{x}\|f\|_{x}=\sup _{x}\left\|f_{x}\right\|_{o}$, where $\|f\|_{x}$ stands for the norm of the image of $f$ in the quotient algebra $A / \bar{J}_{x}$. The type $C$ condition can be written directly:

$$
\|f\|=\sup _{x \in T} \inf _{o \in J}\left\|f_{x}+g\right\| .
$$

Or if we are simply given the ideals $J$ and $\bar{J}$, the type $\mathrm{C}$ norm is identified as the weakest that makes the closure of $J$ no larger than $\bar{J}$. We are of course interested in the topology on $A$, not in the actual norm, and we can if we want modify the type $C$ definition accordingly.

Algebras of type C have been studied extensively in Silov [2]. The C is really a Russian $S$, for "sum." Since the algebra generated by characters is dense, the structure of a type $C$ homogeneous algebra is completely determined by the abstract local algebra $A / \bar{J}$ and by the way in which the characters map into $A / \bar{J}$. For if $e$ is a character then all its translates are scalar multiples of $e$. Specifically, $e_{x}=e\left(x^{-1}\right) e$. Hence if $f$ is a linear combination of characters, $f=c_{1} e_{1}+c_{2} e_{2}+\cdots$, then its norm can be reckoned directly:

$$
\begin{aligned}
\|f\| & =\sup _{x}\left\|f_{x}\right\|_{0}=\sup _{x}\left\|\left(c_{1} e_{1}+c_{2} e_{2}+\cdots\right)_{x}\right\|_{0} \\
& =\sup _{x}\left\|c_{1} e_{1}\left(x^{-1}\right) e_{1}+c_{2} e_{2}\left(x^{-1}\right) e_{2}+\cdots\right\|_{0} .
\end{aligned}
$$


No such simple structure theory is possible for homogeneous algebras not of type $C$. In the absolutely-convergent fourier series, for instance, $A / \bar{J}$ is the complex numbers, so that when $f=\sum c_{k} \exp (i k x)$, its type $C$ norm is $\|f\|$ $=\sup _{x}|f(x)|$, a norm much weaker than the correct norm $\|f\|=\sum\left|c_{k}\right|$.

We shall also require in this section that $A / \bar{J}$ have finite dimension. This requirement can be stated in other ways:

Theorem (Šilov). For a homogeneous algebra A on the torus (or on any compact Lie group) the following three conditions are equivalent:

(1) $A / \bar{J}$ is finite-dimensional, $J$ being the set of all $f \in A$ that vanish on neighborhoods of the identity of the underlying group.

(2) A contains all indefinitely-differentiable functions.

(3) A contains all m-differentiable functions, for some $m$.

Proof. Šilov [1]. Our Lemma B is helpful. Mirkil [1] gives a simple proof of the implication $(2) \rightarrow(3)$.

A typical example of an intrinsic homogeneous algebra of type $\mathrm{C}$, with $A / \bar{J}$ finite-dimensional, is given by the algebra $D_{m}$ of $m$-differentiable functions, i.e. functions having continuous mixed partial derivatives of all orders $\leqq m$. Suppose for simplicity that the underlying torus is one-dimensional. Then $\bar{J}$ is all $m$-differentiable functions $f$ such that $f(o)=f^{\prime}(o)=f^{\prime \prime}(o)=\cdots$ $=f^{(m)}(o)=0$. And $\boldsymbol{A} / J$ is an $(m+1)$-dimensional algebra consisting of all polynomials in a certain nilpotent element $X$ with $X^{m} \neq 0, X^{m+1}=0 . f \in A$ is small if and only if all its derivatives up to the $m$ th are uniformly small. Silov has proved in [1] that on the circle there are no other homogeneous algebras of type C containing $D_{\infty}$. Theorem 3 below generalizes Silov's result.

Theorem 3. Let $A$ be an intrinsic homogeneous algebra of type $\mathrm{C}$ on the $n$-torus $T_{n}$, and let $A$ contain the algebra $D_{\infty}$ of indefinitely-differentiable functions. Then $A$ is for some $m$ exactly the algebra $D_{m}$ of $m$-differentiable functions.

Proof. For simplicity we shall write $D_{\infty}=D$. $D$ has its own natural topology, the only topology that makes it a complete metrisable algebra. A sequence of $f_{k} \in D$ converges to zero in this topology if and only if for each $m$ the sequence of $m$ th derivatives $f_{\boldsymbol{k}}^{(m)}$ converges uniformly to zero. The injection $D \rightarrow A$ is continuous by the closed graph theorem, and $D$ is dense in $A$ because even the algebra generated by characters is dense. Write $\boldsymbol{H}$ for the intersection $J \cap D$, and write $\bar{H}$ for the closure of $H$ in the proper topology of $D . \bar{H}$ is the $f \in D$ that vanish at $o$ together with all derivatives. Since the topology of $D$ is stronger than that induced by $A$, then $H \subset J$ implies $\bar{H} \subset \bar{J}$. Hence there is a natural homomorphism $D / \bar{H} \rightarrow A / \bar{J}$. Since $D$ is dense in $A$, then the image of $D / \bar{H}$ is dense in $A / \bar{J}$. But since $A / \bar{J}$ has finite dimension, the homomorphism $D / \bar{H} \rightarrow A / \bar{J}$ must be onto.

A useful representation of $D / \bar{H}$ can be obtained by taking a coordinate system $x=\left(x_{1}, \cdots, x_{n}\right)$ for $T_{n}$, and choosing $n$ functions $f_{1}, \cdots, f_{n} \in D$ 
which near $o$ imitate the coordinate functions. That is, there exists some neighborhood of $o$ on which the $f_{i}$ are defined by $f_{i}(x)=x_{i}$. Let $X_{i}$ be the image of $f_{i}$ in $\boldsymbol{D} / \overline{\boldsymbol{H}}$. It is not hard to prove that under this representation $\boldsymbol{D} / \overline{\boldsymbol{H}}$ is precisely all formal power series in $X_{1}, \cdots, X_{n}$. And the homomorphism $D \rightarrow D / \bar{H}$ takes every $f \in D$ into its formal maclaurin power series expansion. Automorphisms of the group $T_{n}$ induce unimodular substitutions on the formal power series. It is important to note that the quotient topology $D / \bar{H}$ is different from what we have called the valuation topology on formal power series.

Consider now the ideal $I$ in $D / \bar{H}$ which is the kernel of the homomorphism $\boldsymbol{D} / \overline{\boldsymbol{H}} \rightarrow \boldsymbol{A} / \overline{\boldsymbol{J}}$. We claim that this ideal $\boldsymbol{I}$ is invariant under unimodular substitutions on the formal power series. For suppose $f \in D$ can be approached in the norm of $\boldsymbol{A}$ by $g_{k} \in J$ (i.e. by functions in $\boldsymbol{A}$ vanishing on neighborhoods of $o$ ), and let $L$ be an automorphism of $A$ induced by some automorphism of $T_{n}$. Then $L\left(g_{k}\right) \in J$ (since neighborhoods of $o$ go onto other neighborhoods of $o$ under automorphisms of $\left.\boldsymbol{T}_{n}\right)$, and $L\left(g_{k}\right)$ approaches $L(f)$ in the norm of $\boldsymbol{A}$. Also, $L(f) \in D$. Hence $\bar{J} \cap D$ is invariant under the automorphisms of $\boldsymbol{A}$ induced by automorphisms of $T_{n}$. A similar argument shows that $\bar{H}$ is invariant under these same automorphisms. But $\bar{J} \cap D$ is the kernel of the composite mapping:

$$
D \rightarrow D / \bar{H} \rightarrow A / \bar{J} .
$$

And since the first mapping is onto, the kernel $I$ of the second mapping is $(\bar{J} \cap D) / \bar{H}$. $I$ is clearly invariant, being the quotient of an invariant algebra by an invariant ideal. Hence our claim is established.

By Theorem 2, I must for some $m$ consist of all power series of order $\geqq m$. Hence $A / \bar{J}$ is the commutative algebra generated by $n$ linearly independent elements $Y_{1}, \cdots, Y_{n}$ with the sole rule that all $m_{\text {-fold products are }}$ zero. (Or more strictly, $M / \bar{J}$ is this algebra, and $A / \bar{J}$ is $M / \bar{J}$ with unit adjoined.) Furthermore, under the mapping $A \rightarrow A / \bar{J}$, each $f \in D$ maps onto a truncated version of its maclaurin expansion, all terms of degree $\geqq m$ being set equal to zero. Because $A$ is of type $C$, the above information guarantees that $A$ is exactly $D_{m}$.

6. Counter-examples and remarks. Notice the one-dimensional case in each of our three theorems. Since $T_{1}$ has no oriented automorphisms except the identity, the invariance of $A$ with respect to automorphisms of the underlying group need not be postulated explicitly in Theorem 1 and Theorem 3 . Theorem 2 for formal power series in one variable can omit the hypothesis about unimodular substitutions; the theorem is well known and states that every ideal in $K[[X]]$ is principal with generator of the form $X^{m}$. But when the dimension of the underlying torus is $\geqq 2$, the invariance with respect to automorphisms of $T_{n}$ (or with respect to unimodular substitutions) cannot be omitted. A counter-example to Theorem 1, for instance, could be con- 
structed on the 2-torus by taking the tensor product of a regular algebra with a quasi-analytic algebra. A counter-example to Theorem 3 would be furnished by Silov's vectorially-smooth functions [3] on the 2 -torus. The above counterexample to Theorem 1 makes use of algebras that behave differently on different components of the underlying group. Similar counter-examples for both theorems can be found when the group is a general compact Lie group instead of a torus, even though all automorphisms of the group are admitted. Suppose, for instance, that we have the direct product of two centerless Lie groups. Then each factor is (setwise) invariant under automorphisms of the product group, and we can as before take the tensor product of two algebras that are completely different on the two factors. It might seem that we could sharpen our theorems, since the proofs have used only a restricted kind of unimodular substitution. But the unimodular group is actually generated by substitutions of the form

$$
\begin{array}{ll}
X_{i} \rightarrow X_{i} \pm X_{k}, & i \neq k, \\
X_{j} \rightarrow X_{i}, & j \neq i .
\end{array}
$$

Nonetheless it is likely that Theorem 1 will hold when only rigid automorphisms of $T_{n}$ are used, even though there are examples to show that Theorem 3 is false under these circumstances.

Krull's theorem was an essential link in the proof of Theorem 2. Krull proves in fact that every noetherian local algebra is hausdorff in the valuation topology, and because quotients of local algebras are again local it follows immediately that ideals are closed in the valuation topology. On the other hand the fact that the formal power series are hausdorff in the valuation topology is an easy consequence of our Theorem 2, since the intersection of all powers of the unique maximal ideal is clearly an ideal invariant under unimodular substitutions. It would be interesting to find a simple formulation and proof of Theorem 2 for arbitrary noetherian local algebras.

\section{REFERENCES}

\section{Chevalley}

1. On the theory of local rings, Ann. of Math. vol. 44 (1943) pp. 690-708.

H. Mirkil

G. E. Silov

1. The work of Silov on commutative semi-simple banach algebras, Mathematics Department, University of Chicago, 1952, mimeographed.

1. Homogeneous rings of functions, Amer. Math. Soc. Translation no. 92, from Uspehi Matem. Nauk N.S. vol. 6, no. 1 (41) (1951) pp. 91-137.

2. Rings of type C, Doklady N.S. vol. 66 (1949) pp. 813-816.

3. Vectorially-smooth functions, Uspehi Matem. 'Nauk N.S. vol. 6, no. 5 (45) (1951) pp. 176184.

4. On decomposition of a commutative normed ring in a direct sum of ideals. Mat. Sbornik N.S. vol. 32 (74) (1953) pp. 353-364.

Dartmouth College, HANOVER, N. H. 